\title{
THE ADOMIAN DECOMPOSITION METHOD FOR SOLVING HEAT TRANSFER LIGHTHILL SINGULAR INTEGRAL EQUATION USING MAPLE
}

\author{
* Dalal Adnan Maturi ${ }^{1}$ \\ ${ }^{1}$ Departement of Mathematics, Faculty of Science, King Abdulaziz University, Jeddah, Saudi Arabia \\ * Corresponding Author, Received: 06 Oct. 2021, Revised: 01 Dec. 2021, Accepted: 09 Jan. 2022

\begin{abstract}
In this paper, we study the mathematical physics model, which is an Abel-type Volterra integral equation that describes the distribution Temperature of heat along the surface when the heat transfer to it is balanced by radiation from it. We applied the adomian decomposition method (ADM) to the heat transfer Lighthill Singular integral equation and by converting it to the nonlinear singular Volterra equation of the second kind using the Maple program. The nonlinear term can easily be handled with the help of adomian polynomials. The solution takes the form of a convergent series with easily computable terms. The method is based on the application of Heat Transfer to a nonlinear Integral equation. The Pad e approximants are used effectively in the study to capture the solution's critical behavior. The method's efficiency and reliability are demonstrated by numerical examples. For a broad range of linear and nonlinear singular Integral equations, the approach is very effective and useful in finding analytical and numerical solutions. It gives you more concrete series solutions that converge quickly.
\end{abstract}

Keywords: Adomian Decomposition Method, Heat Transfer, Lighthill Singular Integral Equation, Maple18.

\section{INTRODUCTION}

Many areas of applied mathematics depend on knowledge of integral equations, as they arise naturally in various applications in mathematics, engineering, physics, and technology. They can be used to model a wide range of physical problems such as thermal conductivity, propagation and continuity, mechanics, geophysics, electricity, magnetism, neutron transport, traffic theory, and many more. Integrative equations provide solutions in designing effective parameter algorithms for algebraic curves, surfaces, and superficial surfaces. Many associated elementary and boundary value problems can be reformulated as integral differential equations. Weak singular and scalar integral equations are of particular interest because they are accustomed to solving inverse boundary value problems whose domain is fractal curves, where classical calculus cannot be used. Abel's equations and other fractional integral equations have been extensively studied and are used in the modeling of various phenomena in biophysics, elastic viscosity, electrical circuits, etc. The solubility and properties of Volterra's integral equations have been studied using various analytical and approximate methods.

In this paper, we consider the second kind of nonlinear weakly-singular Volterra integral equations are provided by

$$
\begin{aligned}
u(x)=f(x)+\int_{0}^{x} \frac{\beta}{\sqrt{x-t}} F(u(t)) d t, & \\
x & \in[0, T]
\end{aligned}
$$

$$
\begin{aligned}
& \text { And } \\
& u(x)=f(x)+\int_{0}^{x} \frac{\beta}{[g(x)-g(t)]^{\alpha}} F(u(t)) d t, \\
& 0<\alpha<1, \quad x \in[0, T],
\end{aligned}
$$

where $F(u(t))$ is a nonlinear function of $u(t)$ and $\beta$ is a constant. The generalized nonlinear weakly-singular Volterra equation (2) is also known as the Volterra equation. Many mathematical physics and chemistry applications use these equations, including stereology, heat conduction, crystal formation, and heat radiation from a semiinfinite material. Singular equations with singular kernels include the nonlinear weakly-singular and extended nonlinear weakly-singular equations (1) and $(2)$

$$
\begin{gathered}
K(x, t)=\frac{1}{\sqrt{x-t}}, \\
K(x, t)=\frac{1}{[g(x)-g(t)]^{\alpha}}, \quad 0<\alpha<1,
\end{gathered}
$$

Several authors have investigated the numerical solvability of (1). In [1], Diogo et al. used a collocation with graded mesh. A hybrid collocation approach was introduced in [2] for (1). After a smoothing adjustment, a Nystrom type technique [3] was also considered (1).

The Jacobi spectral collocation method [4] was recently proposed for the solution of (1). Ortiz and Samara [5] introduced an operational strategy based on the conventional Tau approach for the numerical solution of nonlinear ordinary differential equations 
with certainly added constraints in 1981. Ordinary differential equations (ODEs), partial differential equations, integral equations, and integrodifferential equations have all been solved using the Tau technique $[8,7,6]$.

The kernels in many applications modeled by integral equations are not smooth, making it difficult to identify a solution and numerically approximate it, as the convergence of approximation methods is dependent on the smoothness of the solution in general. As a result, traditional analytical approaches, such as projection methods, perform poorly in such situations, as the linear system they lead to is often poorly conditioned and difficult to solve. When classical calculus cannot be utilized, proving convergence and estimating error can be time-consuming. They frequently have a significant implementation cost. As a result, fast, easy-to-use numerical algorithms for these types of equations are in high demand. The approach we offer is based on a traditional fixedpoint solution that has been appropriately altered. The product integration numerical approach we utilize is also highly efficient for approximating the integrals involved, because most of the computations may be done in a single step.

The kernels in many applications modeled by integral equations are not smooth, making it difficult to identify a solution and numerically approximate it, as the convergence of approximation methods is dependent on the smoothness of the solution in general. As a result, traditional analytical approaches, such as projection methods, perform poorly in such situations, as the linear system that they lead to is often poorly conditioned and difficult to solve.

When classical calculus cannot be utilized, proving convergence and estimating error can be time-consuming. They frequently have a significant implementation cost. As a result, fast, easy-to-use numerical algorithms for these types of equations are in high demand.

The adomian decomposition approximation of the second kind weakly singular nonlinear VolterraHammerstein integral equations is the subject of this research.

\section{RESEARCH SIGNIFICANCE}

Integral equations emerge naturally in many applications in Mathematics, Engineering, Physics, and Technology, hence they are used in many domains of Applied Mathematics. They can be used to simulate a variety of physical problems, including heat conduction, diffusion, and continuum. Mechanics, geophysics, electricity, magnetism, neutron transport, traffic theory, and many other subjects are covered in this paper. Integral equations help in the development of efficient algebraic parametrization techniques curves, surfaces, and hypersurfaces are all examples of curves. There are numerous initial and boundary value concerns related to Integral equations that can be reformed from ordinary and partial differential equations Integral equations, both singular and weakly singular, are of particular significance since they are used to handle inverse boundary value problems using fractal curves as domains, using classical calculus unable cannot be utilized The Abel equations, as well as other fractional order integral equations, were widely researched and are employed in the simulation of biology, viscoelasticity, electrical circuits, and other phenomena.

In this research, we shed light on the heat transfer Lighthill singular integral equation and we solved it with the Maple program to know the properties of this equation, and then deduce its properties and advantages that are useful for engineers, mathematicians, and physicists on a large scale.

\section{THE ADOMIAN DECOMPOSITION METHOD}

Consider the differential equation [11]. Because Eq. (1) is a particular case of the generalized nonlinear weakly-singular Volterra equation (2), the Adomian decomposition method will be used to solve it. $\alpha=12, g(x)=x$.As previously said, we will provide a brief overview of the method's framework. We substitute the decomposition series for the decomposition series to (2) find the solution $u(x)$ of $u(x)=\sum_{n=0}^{\infty} u_{n}(x)$

$$
u(x)=\sum_{n=0}^{\infty} u_{n}(x)
$$

$$
\begin{aligned}
& \text { And } \\
& \qquad F(u(x))=\sum_{n=0}^{\infty} A_{n}(x) \\
& A_{n}=\frac{1}{n !} \frac{d^{n}}{d \lambda^{n}}\left[F\left(\sum_{n=0}^{\infty} \lambda^{i} u_{i}\right)\right]_{\lambda=0}, n=0,1,2, \cdots
\end{aligned}
$$

where an is the Adomian polynomials, and (2) on both sides to get

$$
\begin{aligned}
& \sum_{n=0}^{\infty} u_{n}(x) \\
& =f(x)+\int_{0}^{x} \frac{\beta}{[g(x)-g(t)]^{\alpha}}\left(\sum_{n=0}^{\infty} A_{n}(t)\right) d t, \\
& 0<\alpha<1,
\end{aligned}
$$

$$
u_{0}(x)=f(x)
$$




$$
\begin{aligned}
& u_{1}(x)=\int_{0}^{x} \frac{\beta}{[g(x)-g(t)]^{\alpha}} A_{0}(t) d t, \\
& u_{2}(x)=\int_{0}^{x} \frac{\beta}{[g(x)-g(t)]^{\alpha}} A_{1}(t) d t, \\
& u_{3}(x)=\int_{0}^{x} \frac{\beta}{[g(x)-g(t)]^{\alpha}} A_{2}(t) d t, \\
& u_{4}(x)=\int_{0}^{x} \frac{\beta}{[g(x)-g(t)]^{\alpha}} A_{3}(t) d t,
\end{aligned}
$$

and so forth After determining te components $u 0(x), u 1(x)$, and $u 2(x)$, the solution $u(x)$ of (2) will be determined as a series by substituting the (4) derived components in the formula.

\section{Definition 1.}

Let $(X,\|\cdot\|)$ be a Banach space. A mapping $T$ : $X \rightarrow X$ is called a $q$-contraction if there exists a constant $0 \leq q<1$ such that

$$
|| T x-T y\|\leq q|| x-y\|,
$$

for all $x, y \in X$. On Banach spaces, the wellknown contraction principle holds:

\section{Theorem 1.}

Consider a Banach space $(X,\|\cdot\|)$ and let

$T: X \rightarrow X$ be a q-contraction. Then

(i) $T$ has exactly one fixed point, which means equation $x=T x$ has exactly one solution $x^{*} \in$ $X$;

(ii) the sequence of successive approximations $x_{n+1}=T x_{n}, n \in N$, converges to the solution $x^{*}$, where $x_{0}$ can be any arbitrary point in $X$;

(iii) for every $n \in N$, the following error estimate $\left\|x_{n}-x^{*}\right\| \leq \frac{q_{n}}{1-q}\left\|T x_{0}-x_{0}\right\|$ holds.

We utilize Banach's theorem to prove the existence and uniqueness of a solution to Equation (1) under particular conditions, and to approximate it by applying the operator consecutively. The values of the solution at specific nodes are then approximated using an appropriate numerical integration scheme.

Remark1. Theorem 1remains valid when $X$ is replaced by a closed subset $\boldsymbol{Y} \subseteq \boldsymbol{X}$, satisfying $\boldsymbol{T}(\boldsymbol{Y}) \subseteq \boldsymbol{Y}$.

Remark 2. Since $\boldsymbol{a} \in \boldsymbol{C}([\mathbf{0}, \boldsymbol{T}] \times[0, T] \times$ $\boldsymbol{R})$ and $\boldsymbol{f} \in \boldsymbol{C}(\mathbf{0}, \boldsymbol{T}]$, it is well known that the operator $\boldsymbol{F}: \boldsymbol{C}[\mathbf{0}, \boldsymbol{T}] \rightarrow \boldsymbol{C}[\mathbf{0}, \boldsymbol{T}]$ is well defined, i.e., $\boldsymbol{F}(\boldsymbol{C}[\mathbf{0}, \boldsymbol{T}]) \subseteq \boldsymbol{C}[\mathbf{0}, \boldsymbol{T}])$

Theorem 2. Let $\boldsymbol{F}:(\boldsymbol{X},\|\cdot\| \boldsymbol{\tau}) \rightarrow(\boldsymbol{X},\|\cdot\| \boldsymbol{\tau})$

be defined by Equation (3). Assume that there exists a constant $\boldsymbol{L}>\mathbf{0}$ such that

$$
|a(t, s, u)-a(t, s, v)| \leq L|u-v|,
$$

for all $\boldsymbol{t}, \boldsymbol{s} \in[\mathbf{0}, \boldsymbol{T}]$ and all $\boldsymbol{u}, \boldsymbol{v} \in \boldsymbol{R}$. Then (i) Equation (4) has a unique solution $\boldsymbol{u} * \in \boldsymbol{X}$;

(ii) the sequence of successive approximations

$$
u_{n+1}=F u_{n+1} n=0,1, \cdots
$$

converges to the solution $\mathrm{u} *$ for any $\boldsymbol{u} \mathbf{0} \in \boldsymbol{X}$;

(iii) for every $n \in N$, the following error estimate

$$
\|u n-u *\| \tau \leq q n 1-q\|F u 0-u 0\| \tau
$$

holds, where $\boldsymbol{q}=\frac{\boldsymbol{L} \Gamma(\boldsymbol{\alpha})}{\boldsymbol{\tau}^{\boldsymbol{\alpha}}}$ is the contraction constant

\section{Theorem 3.}

Let the conditions of Theorem 2 hold If, in addition, $\boldsymbol{f} \in \boldsymbol{C} \mathbf{2}, \mathbf{1}-\boldsymbol{\alpha}(\mathbf{0}, \boldsymbol{T}]$ and

$$
a \in C 2([0, T] \times[0, T] \times R),
$$

then $\boldsymbol{u} * \in \boldsymbol{C} \mathbf{2}, \mathbf{1}-\boldsymbol{\alpha}(\mathbf{0}, \boldsymbol{T}]$,

The Lipschitz condition in Theorem 2 can be very prohibitive if required on the entire space. To be able to use it on a wider range of applications, we restrict it to a closed subset. Let || · || denote the Chebyshev norm on $\boldsymbol{C}[\mathbf{0}, \boldsymbol{T}]$ (which is equivalent to the Bielecki norm) and consider the closed ball

$$
B R:=\{u \in C[0, T]\|u-f\| \leq R\},
$$

for some $\boldsymbol{R}>\mathbf{0}$. Then $\boldsymbol{B} \boldsymbol{R} \subseteq \boldsymbol{X}$ and we have the following results

\section{Theorem 4.}

Let us suppose that there exists a constant $\boldsymbol{L}>\mathbf{0}$ such that

$$
|a(t, s, u)-a(t, s, v)| \leq L|u-v|,
$$

for all $\boldsymbol{t}, \boldsymbol{s} \in[\mathbf{0}, \boldsymbol{T}]$ and all

$$
u, v \in\left[R_{1}-R, R_{2}+R\right],
$$

where $R_{1}:=\min t \in[0, T] f(t), R_{2}:=$ $\max t \in[0, T] \boldsymbol{f}(\boldsymbol{t})$. Further assume that

$$
\frac{M T^{\alpha}}{\alpha} \leq R,
$$

where $\boldsymbol{M}:=\max |\boldsymbol{a}(\boldsymbol{t}, \boldsymbol{s}, \boldsymbol{u})|$ over all $\boldsymbol{t}, \boldsymbol{s} \in$ $[\mathbf{0}, \boldsymbol{T}]$ and all $\boldsymbol{u}, \boldsymbol{v} \in[\boldsymbol{R} \mathbf{1}-\boldsymbol{R}, \boldsymbol{R} \mathbf{2}+\boldsymbol{R}]$. Then the conclusions of Theorem 2 hold on $\boldsymbol{B}_{\boldsymbol{R}}$.

\section{SEVERAL EXAMPLE}

In this section, we use the adomian decomposition method (ADM) to solve the problems discussed earlier. In addition, the number of iterations for various values is also determined. 
And also plot the surface for the approximate solution of the heat transfer Lighthill singular integral equation.

Example1. Consider the heat transfer Lighthill Singular integral equation

$$
\begin{gathered}
u(x)=1-\frac{\sqrt{3}}{\pi} \int_{0}^{x} \frac{t^{\frac{1}{3}} u^{4}(t)}{(x-t)^{\frac{2}{3}}} d t, \\
x \in[0,1]
\end{gathered}
$$

With the exact solution $u^{*}(x)=\sqrt{x}$

Applying the Adomian Decomposition Method using Maple, we find

Table 1 Numerical results and exact solution of heat transfer Lighthill Singular integral equation for example 1

\begin{tabular}{cccc}
\hline $\boldsymbol{x}$ & $\begin{array}{c}\text { Exact } \\
=\sqrt{\boldsymbol{x}}\end{array}$ & $\boldsymbol{u}(\boldsymbol{x})$ & Error \\
\hline 0.0010 & 0.0316228 & 0.0316227 & 0.0000001 \\
0.0020 & 0.0447214 & 0.0447209 & 0.0000005 \\
0.0030 & 0.0547723 & 0.0547710 & 0.0000013 \\
0.0040 & 0.0632456 & 0.0632429 & 0.0000027 \\
0.0050 & 0.0707107 & 0.0707060 & 0.0000047 \\
0.0060 & 0.0774597 & 0.0774523 & 0.0000073 \\
0.0070 & 0.0836660 & 0.1475523 & 0.0000108 \\
0.0080 & 0.0894427 & 0.0894277 & 0.0000151 \\
0.0090 & 0.0948683 & 0.0948481 & 0.0000202 \\
0.0100 & 0.1000000 & 0.0999737 & 0.0000263 \\
\hline
\end{tabular}

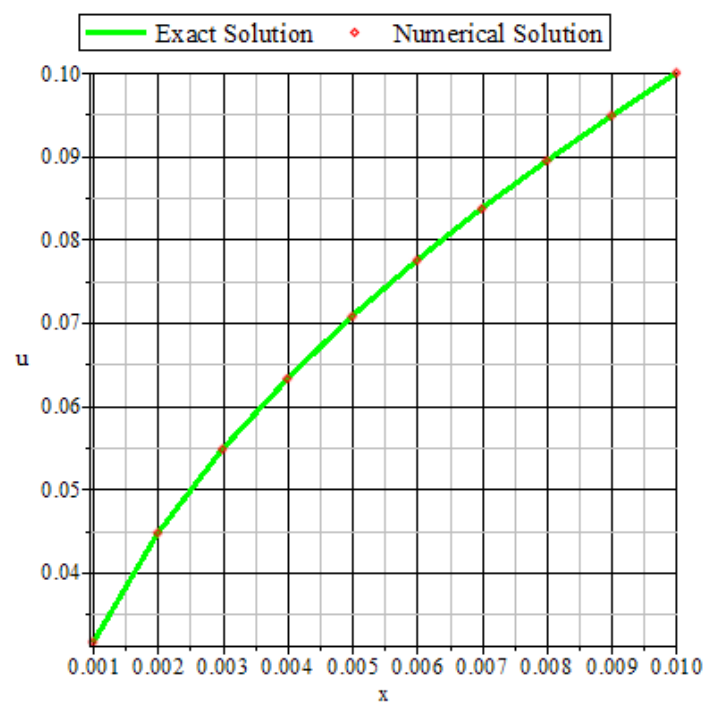

Fig.1 Plot 2D of the exact solutions result of heat transfer Lighthill Singular integral equation for example 1.

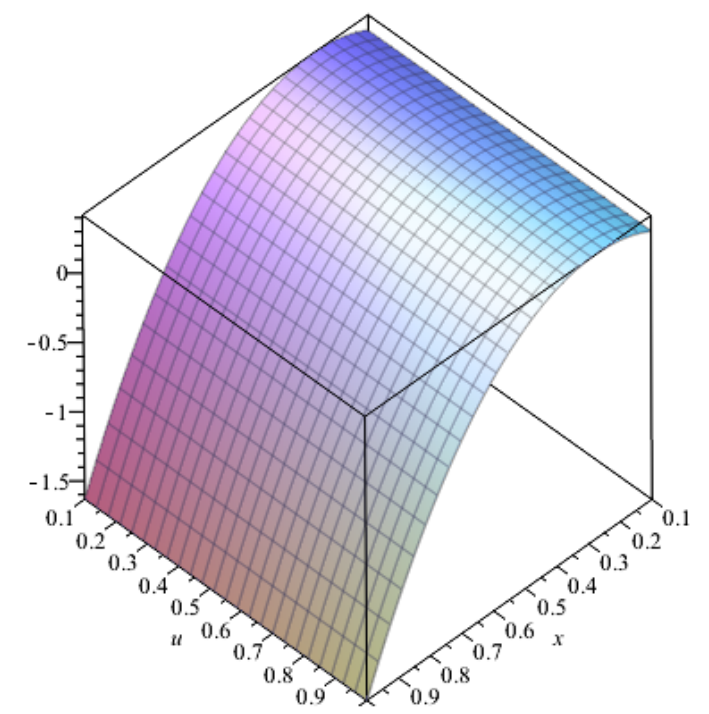

Fig.2 The surface shows the approximate solution of the heat transfer Lighthill Singular integral equation for example 1 .

Example2. Consider the heat transfer Lighthill Singular integral equation

$$
\begin{gathered}
u(x)=x+\frac{4}{3} x^{\frac{3}{2}}-\int_{0}^{x} \frac{1}{\sqrt{x-t}} u(t) d t \\
x \in[0,1]
\end{gathered}
$$

With the exact solution $u^{*}(x)=x$

Applying the Adomian Decomposition Method using Maple, we find

Table 2 Numerical results and exact solution of heat transfer Lighthill Singular integral equation for example 2

\begin{tabular}{cccc}
\hline $\boldsymbol{x}$ & $\begin{array}{c}\text { Exact } \\
=\boldsymbol{x}\end{array}$ & $\boldsymbol{u}(\boldsymbol{x})$ & Error \\
\hline 0.0010 & 0.0010000 & 0.0010001 & 0.0000001 \\
0.0020 & 0.0020000 & 0.0020003 & 0.0000003 \\
0.0030 & 0.0030000 & 0.0030008 & 0.0000008 \\
0.0040 & 0.0040000 & 0.0040017 & 0.0000017 \\
0.0050 & 0.0050000 & 0.0050030 & 0.0000030 \\
0.0060 & 0.0060000 & 0.0060047 & 0.0000047 \\
0.0070 & 0.0070000 & 0.0070069 & 0.0000069 \\
0.0080 & 0.0080000 & 0.0080096 & 0.0000096 \\
0.0090 & 0.0090000 & 0.0090129 & 0.0000129 \\
0.0100 & 0.0100000 & 0.0100168 & 0.0000168 \\
\hline
\end{tabular}




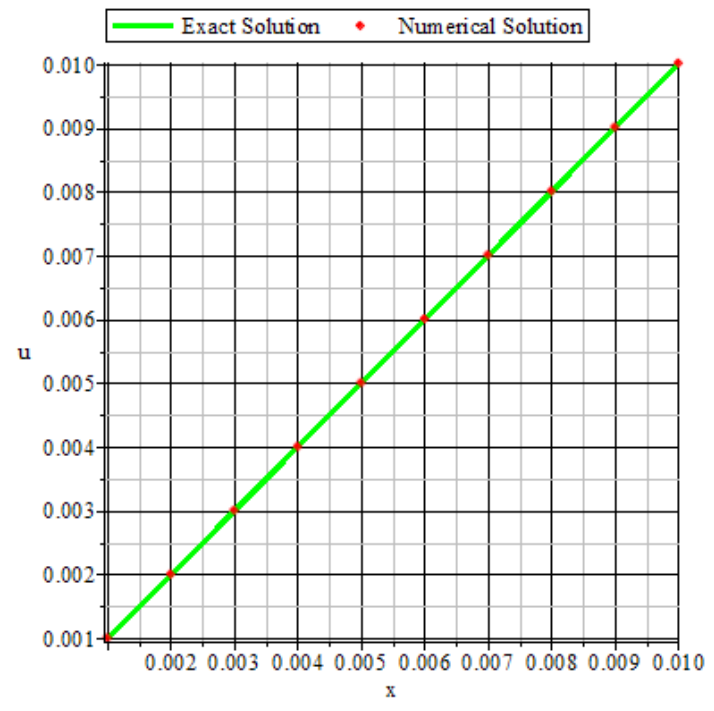

Fig.3 Plot 2D of the exact solutions result of heat transfer Lighthill Singular integral equation for example 2.

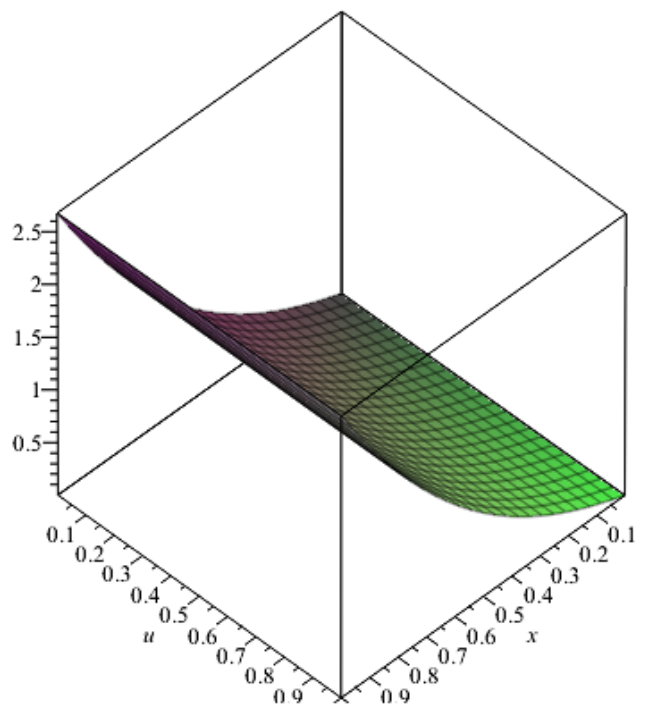

Fig.4 The surface shows the approximate solution of the heat transfer Lighthill Singular integral equation for example 2.

Example3. Consider the heat transfer Lighthill Singular integral equation

$$
u(x)=2 \sqrt{x}-\int_{\substack{0 \\ x \in[0,2]}}^{x} \frac{1}{\sqrt{x-t}} u(t) d t
$$

With the exact solution

$$
u^{*}(x)=1-e^{\pi x} \operatorname{erfc}(\sqrt{\pi x})
$$

Applying the Adomian Decomposition Method using Maple, we find

Table 3 Numerical results and exact solution of heat transfer Lighthill Singular integral equation for example 3

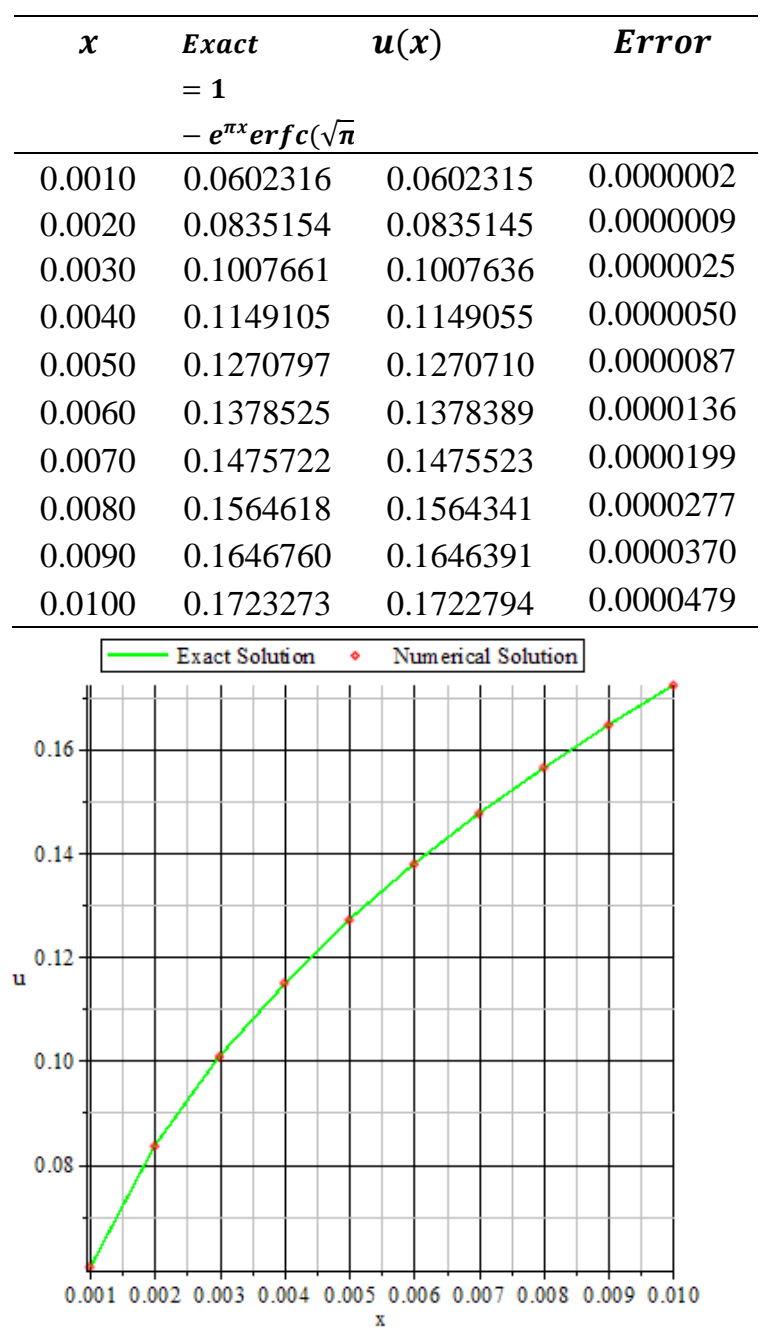

Fig.5 Plot 2D of the exact solutions result of heat transfer Lighthill Singular integral equation for example3.

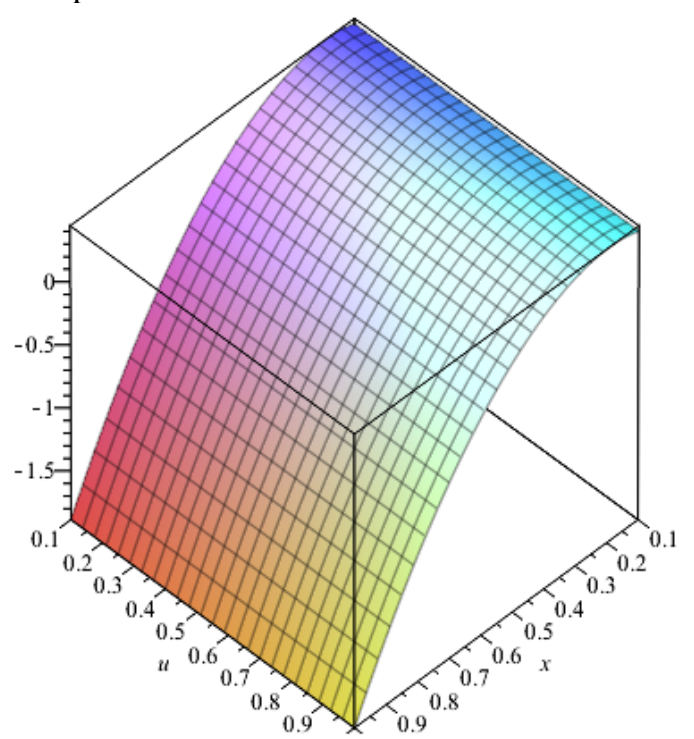

Fig.6 The surface shows the approximate solution of the heat transfer Lighthill Singular integral equation for example 3 . 
Example4. Consider the heat transfer Lighthill Singular integral equation

$$
u(x)=x-\frac{1}{\sqrt{\pi}} \int_{0}^{x} \frac{1}{\sqrt{x-t}} u^{3}(t) d t
$$

With the exact solution

$$
\boldsymbol{u}^{*}(\boldsymbol{x})=\boldsymbol{x}
$$

Applying the Adomian Decomposition Method using Maple, we find

Table 4 Numerical results and exact solution of heat transfer Lighthill Singular integral equation for example 4

\begin{tabular}{cccc}
\hline $\boldsymbol{x}$ & Exact $=\boldsymbol{x}$ & $\boldsymbol{u}(\boldsymbol{x})$ & Error \\
\hline 0.0010 & 0.0100000 & 0.0099999 & 0.0000001 \\
0.0020 & 0.0200000 & 0.0199994 & 0.0000006 \\
0.0030 & 0.0300000 & 0.0299976 & 0.0000024 \\
0.0040 & 0.0400000 & 0.0399934 & 0.0000066 \\
0.0050 & 0.0500000 & 0.0499856 & 0.0000144 \\
0.0060 & 0.0600000 & 0.0599727 & 0.0000273 \\
0.0070 & 0.0700000 & 0.0699533 & 0.0000467 \\
0.0080 & 0.0800000 & 0.0799255 & 0.0000745 \\
0.0090 & 0.0900000 & 0.0898875 & 0.0001125 \\
0.0100 & 0.1000000 & 0.0998375 & 0.0001625 \\
\hline
\end{tabular}

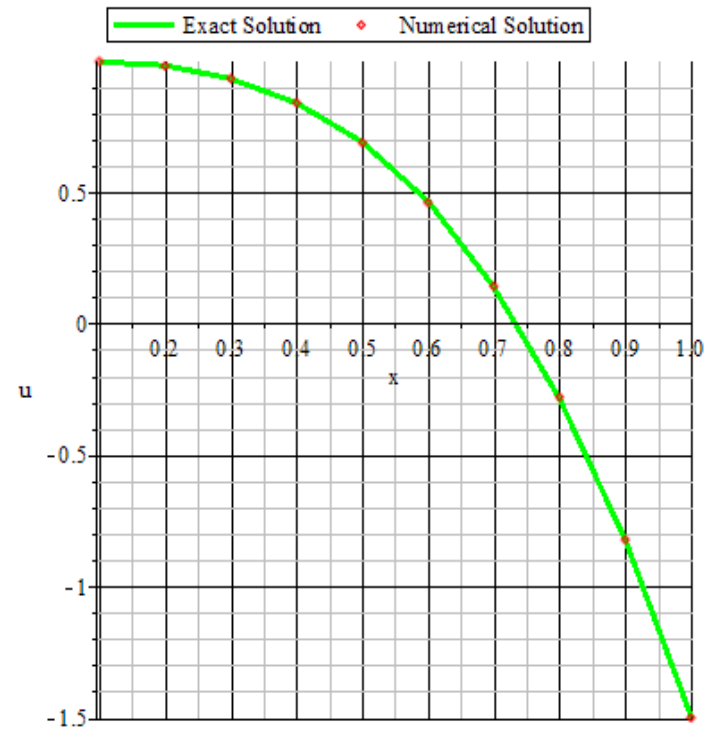

Fig.7 Plot 2D of the exact solutions result of heat transfer Lighthill Singular integral equation for example 4.

\section{CONCLUSION}

In this paper, we are frequently directed to integral Volterra equations that are difficult to solve analytically when addressing numerous nonlinear issues in thermal conductivity, boundary layer heat transfer, chemical kinetics, and superfluidity.

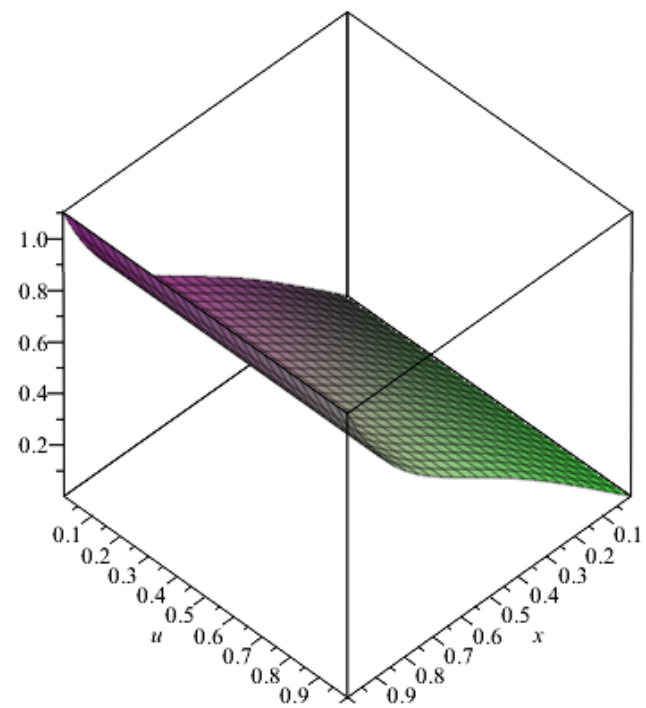

Fig.8 The surface shows the approximate solution of the heat transfer Lighthill Singular integral equation for example 4.

\section{CONCLUSION}

In this paper, we are frequently directed to integral Volterra equations that are difficult to solve analytically when addressing numerous nonlinear issues in thermal conductivity, boundary layer heat transfer, chemical kinetics, and superfluidity. The domain decomposition method is provided in this article for solving nonlinear Volterra integral equations with a single weak core. The Maple program was used to produce numerical results as well as drawings illustrating the degree of accuracy between the numerical and accurate solutions. Some examples are given to demonstrate the scheme's applicability and accuracy. Other numerical methods can be used in the future, and the results of the numerical solution can be compared to the exact solution.

Some examples are given to demonstrate the scheme's applicability and accuracy. We can apply different numerical methods in the future, and we may extend the current method to a system of nonlinear Volterra integral equations and nonlinear Volterra integral equations of the mix type or the first type by comparing the results of the numerical solution with the exact solution.

\section{ACKNOWLEDGMENTS}

This paper was supported by the Deanship of Scientific Research (DSR), King Abdulaziz University, Jeddah.

\section{REFERENCES}

[1] Diogo M. T., Rebelo M. S., Fully discretized 
collocation methods for nonlinear singular Volterra integral equations, Journal of Computational and Applied Mathematics, Vol.247,2013, pp.84-101.

[2] Rebelo M. S., Diogo M. T., A hybrid collocation method for a nonlinear Volterra integral equation with the weakly singular kernel, Journal of Computational and Applied Mathematics, Vol. 234,2010, pp.2859-2869.

[3] Baratella P., A Nystrom interpolant for some weakly singular nonlinear Volterra integral equations Journal of Computational and Applied Mathematics, Vol.237,2013, pp.542555.

[4] Allaei S. S., Diogo M. T., Rebelo M. S., The Jacobi collocation method for a class of nonlinear Volterra integral equations with the weakly singular kernel, Journal of Scientific Computing Volume 69, Issue2, 2016, pp.673695.

[5] Ortiz L. E., Samara H., An operational approach to the Tau method for the numerical solution of nonlinear differential equations, Applied Mathematics and Computing, Vol.27,1981, pp.15-25.

[6] Ebadi M. G., Rahimi-Ardabili Y. M., Shahmorad S., Numerical solution of the nonlinear Volterra integro-differential equations by the Tau method, Applied Mathematics, and Computing, Vol.188, Issue 2 2007, pp.1580-1586.

[7] Ghoreishi F., Hadizadeh M., Numerical computation of the Tau approximation for the Volterra-Hammerstein integral equations, Numer. Algor, Vol.52,2009, pp.541-559.

[8] Hossieni M. S., Shahmorad S., Tau numerical solution of Fredholm integrodifferential equations with arbitrary polynomial bases, Appl. Math. Model, Vol. 27, 2003, pp. 145-154.

[9] Adomian G., Solving Frontier Problems of Physics: The Decomposition Method, Kluwer Academic Publishers, Dordrecht,1994.

[10] Wazwaz M. A., The variational iteration method; a reliable tool for solving linear and nonlinear wave equations, Computers and Mathematics with Applications, Vol. 54,2007, pp.926-932.

[11] Wazwaz M. A., A First Course in Integral Equations, World Scientific, Singapore, 1997.

[12] Wazwaz M. A., Khuri A. S., The variational iteration method for solving the Volterra integrodifferential forms of the lane-Emden and the Emden-Fowler problems with initial and boundary value conditions, DE GRUYTER Open Eng. Vol.5, 2015,pp.31-41.

[13] He H. J., Homotopy perturbation technique, Computer Methods in Applied Mechanics and engineering. 178, 1999, pp. 257-262.

[14] He H. J., A coupling method of a homotopy technique and a perturbation technique for nonlinear problems, International Journal of NonLinear Mechanics, Vol. 35, 2000, pp. 37-43.

[15] He H. J., Homotopy perturbation method: anew non-linear analytical technique, Applied Mathematics, and Computation, Vol. 135, 2003, pp.73-79.

[16] He H. J., Asymptotology by a homotopy perturbation method, Applied Mathematics, and Computation. 156, 2004, pp. 591-596.

[17] He H. J., Application of homotopy perturbation method to nonlinear wave equations, Chaos, Solitons and Fractals, Vol. 26, 2005, pp. 695700 .

[18] He H. J., Homotopy perturbation method for solving boundary value problems, Physics Letters A, Vol. 350, 2006, pp. 87-88.

[19] He H. J., "New interpretation of homotopy perturbation method", International Journal of Modern Physics B, Vol. 18, 2006, pp. 25612568.

[20]Balakumar V., Murugesan K., Numerical Solution of Systems of Linear Volterra Integral Equations Using Block-Pulse Functions, Malaya Journal of Matematik, Vol.1,2013, pp.77-84.

[21] Becker C. L., and M. Wheeler, Numerical and Graphical Solutions of Volterra Equations of the Second Kind, Maple Application Center, 2005.

[22] Burden L. R., and Douglas J. F., Numerical Analysis, 8th ed., Thomson Brooks/Cole, Belmont, CA, 2005.

[23] T. A. Burton, Volterra Integral and Differential Equations, 2nd ed., Mathematics in Science \& Engineering, 202, Elsevier, 2005.

[24] Berenguer I. M., Gamez D., Garralda-GuillemI. A. I., Ruiz Galan M.., and M.C. SerranoPerez C. M., Biorthogonal Systems for Solving Volterra Integral equation Systems of the Second Kind, Journal of Computational and Applied Mathematics, Vol.235, Issue 7 2011, pp.1875-1883.

[25] Biazar J., and Eslami M., Modified HPM for Solving Systems of Volterra Integral Equation of the Second Kind, Journal of King Saud University-Science, Vol.23, Issue 1,2011, pp.35-39.

[26] Linz P., Analytical and Numerical Methods for Volterra Equations, Studies in Applied Mathematics 7, SIAM, Philadelphia, 1985.

[27] Linz P., A simple approximation method for solving Volterra integro-differential equations of the first kind, J. Inst. Math. Appl., Vol.14,1974, pp.211-215.

[28] Mirzaee F., Numerical Computational Solution of the Linear Volterra Integral Equations System Via Rationalized Haar Functions, Journal of King Saud University- Science, 
Vol.22, Issue 4, 2010, pp.265-268.

[29] Wazwaz M. A., Linear and Nonlinear Integral Equation: Methods and Applications, Springer, 2011.

[30]Effati S., and NooriSkandari H.M., Optimal Control Approach for Solving Linear Volterra Integral Equations, I.J. Intelligent Systems, and Applications, 2012,4, pp.40-46.

[31] Maturi A. D., Numerical Solution of System of Two Nonlinear Volterra Integral Equations, International Journal of Computers \& Technology, Vol12, No.10,2014, pp.39673975.

[32] Maturi A. D., Bajamal Z. A., AlGethami M.B., Numerical Solution of Volterra Integral Equation of Second Kind Using Implicit Trapezoidal, Journal of Advances In Mathematics, Vol8, No.2, 2014, pp.1540-1553.

[33] Maturi A. D., Adomian Decomposition Method of Fredholm Integral Equation of the Second Kind Using Maple, Journal of Advances In Mathematics, Vol9, No.1, 2014, pp.1868-1875.

[34] Maturi A. D., Application of Adomian Decomposition Method for Solving of Fredholm Integral Equation of the Second Kind, European Journal of Science and Engineering, Vol9, No.2, 2014, pp.1-9.

[35] Sorkin H. H., S. Yalcinbas S., Approximate solutions of linear Volterra integral equation systems with variable coefficients, Applied Mathematical Modeling, Vol.34,2010, pp. 3451-3464.
Solution of System of Three Nonlinear Volterra Integral Equation Using Implicit Trapezoidal, Journal of Mathematics Research; Vol. 10, No. 1, 2018, pp.1916-9795.

[37] Maturi A. D., The Modified Decomposition Method For Solving Volterra Integral Equation Of The Second Kind Using Maple, International Journal of GEOMATE, Vol.17, Issue 62, 2019, pp. 23-28.

[38] Maturi A. D., Aljedani I. A., Alaidarous S. E., Finite Difference Method For Solving Heat Conduction Equation Of The Granite, International Journal of GEOMATE, Vol.17, Issue 61, 2019, pp. 135 -140.

[39] Maturi A. D., Finite Difference Method For Solving Heat Conduction Equation Of The Brick, International Journal of GEOMATE, Vol.18, Issue 68, 2020, pp. 114-119.

[40] Maturi A. D., Simbawa A. E., The Modified Decomposition Method For Solving Volterra Fredholm Integro-Differential Equation Using Maple, International Journal of GEOMATE, Vol.18, Issue 67, 2020, pp. 84-89.

[41] Micula S., A Numerical Method for Weakly Singular Nonlinear Volterra Integral Equations of the Second Kind, Symmetry, Vol.12, 2020, Issue 1862, pp.1-15.

Copyright (C) Int. J. of GEOMATE All rights reserved, including making copies unless permission is obtained from the copyright proprietors. 